\title{
Manuel anatomique de dissection de la base du crâne
}

\author{
Giovanni BRIGANTI * \\ Département de Physiopathologie, École Supérieure de la Santé
}

February 17, 2019

\section{Neuro-ostéo-anatomie de la base du crâne}

\subsection{Face interne de la base du crâne}

La face interne de la base du crâne comprend 3 grandes dépressions qui correspondent à 3 niveaux différents : les fosses crâniennes antérieure, moyenne et postérieure.

Fosse crânienne antérieure La fosse crânienne antérieure est formée par l'os frontal en avant, l'os ethmoïde médialement et le corps et petites ailes de l'os sphénoïde postérieurement.

Os frontal La plus grande partie de cette fosse est occupée par la partie orbitaire de l'os frontal. Cette partie porte les lobes frontaux et constitue le toit de l'orbite. Il existe une saillie osseuse médiane de l'os frontal : la crête frontale. A sa base se trouve le foramen caecum.

Os ethmoïde La cristagalli est une crête osseuse médiane appartenant à l'os ethmoïde et située en arrière du foramen caecum. De chaque côté de la cristagalli se trouve la lame criblée de l'os ethmoïde. Cette dernière a l'aspect d'une passoire et livre passage à des radicelles nerveuses du nerf I olfactif. Toujours dans la lame criblée, se trouvent les foramina ethmoïdaux antérieurs et postérieurs livrant passage à des branches homonymes du V1 et se prolongeant dans la partie ethmoïdale de l'orbite oculaire.

*giovanni.briganti@hotmail.com 
Os sphénoïde Les petites ailes de l'os sphénoïde présentent un prolongement médian et postérieur : les processus clinoïdes antérieurs. Entre les 2 petites ailes se trouve le limbus sphénoïdal.

Orbite oculaire L'orbite oculaire est la poche osseuse contenant le globe oculaire ainsi que les muscles, vaisseaux et nerfs assurant sa fonction. La paroi antérieure de l'orbite ou toit est constituée par la partie horizontale de l'os frontal. La paroi médiale est constituée supérieurement par l'os frontal, l'os maxillaire et lacrymal antérieurement, et l'os ethmoïde postérieurement. L'os lacrymal présente la crête lacrymale externe et interne (ou antérieure et postérieure), deux reliefs osseux séparés par une fosse lacrymale (ou sillon lacrymal) ou s'abouche le canal ou conduit lacrymal pour le larmoiement. La paroi latérale est constituée par l'os zygomatique antérieurement et par la grande aile de l'os sphénoïde (se prolongeant dans la paroi postérieure de l'orbite). La grande aile du sphénoïde présente la fissure orbitaire supérieure, foramen livrant passage aux nerfs III (oculomoteur commun), IV (trochléaire), VI (oculomoteur externe ou abducens), V1 (ophtalmique) et la veine ophtalmique supérieure. La veine ophtalmique inférieure passe dans la fissure orbitaire inférieure. La petite aile du sphénoïde, médiale à la grande aile du sphénoïde, présente le canal optique, foramen livrant passage au nerf II optique pour la vision ainsi qu'à l'artère ophtalmique innervant l'œil.

\section{Fosse crânienne moyenne}

Os sphénoïde Sa forme évoque celle d'un papillon. Elle présente un relief en forme de selle, la selle turcique, située à la face sup du corps de l'os sphénoïde. Celle-ci est flanquée de deux dépressions latérales où viendront se loger les lobes temporaux du cerveau. Ces dépressions latérales sont constituées ventro-médialement par les grandes ailes de l'os sphénoïde, ventrolatéralement par l'écaille du temporal (partie squameuse) et dorsalement par le rocher (partie pétreuse du temporal). Les limites de la fosse crânienne moyennes sont antéro-latéralement le bord tranchant des petites ailes de l'os sphénoïde, antéro-médialement le limbus sphénoïdal (bord antérieur du sillon pré chiasmatique, lieu de réunion entre les deux nerfs optiques, se séparant en suite par deux bandelettes optiques, une gauche et une droite, allant dans le cortex occipital pour délivrer l'information visuelle), postérolatéralement la partie pétreuse des os temporaux et postéro-médialement le dorsum sellae. La selle turcique est bordée par les processus clinoïdes antérieurs et postérieurs et comprend 3 parties : le tubercule de la selle (situé en avant de la fosse hypophysaire), la fosse hypophysaire où se loge l'hypophyse et le dos de la selle (dorsum sellae). De chaque côté de la base du corps de l'os sphénoïde, quatre foramina disposés en croissant tra- 
versent la grande aile du sphénoïde : fissure orbitaire supérieure, foramen rond, foramen ovale, foramen épineux. La fissure orbitaire supérieure sépare la grande de la petite aile. Elle établit une communication entre l'orbite et la fosse crânienne moyenne. Elle livre passage aux nerf crâniens oculomoteurs III, IV, VI, le nerf ophtalmique V1 et à la veine ophtalmique supérieure. Le foramen rond est situé dorsalement par rapport à l'extrémité médiale de la fissure orbitaire supérieure. Il livre passage au nerf V2 maxillaire (sensibilité du tiers moyen de la face, muqueuse du pharynx via nerf pharyngien, dure mère via nerf méningé, cellules éthmoïdales, muqueuse nasale via rameau nasal et naso-palatin, voute palatine via nerfs grand et petits palatins ainsi que alvéoles dentaires). Le foramen ovale est plus grand que le foramen rond et se positionne postéro latéralement par rapport à celui-ci. Il s'ouvre dans la fosse infra temporale et livre passage au nerf V3 mandibulaire donnant l'innervation sensitive du tiers inférieur de la face, les méninges de la fosse cérébrale moyenne, la mandibule et les dents inférieures, ainsi que l'innervation motrice de certains muscles du plancher buccal (ou sus-hyoïdiens) ainsi que des muscles de la mastication (masséter, temporal, ptérygoïdiens médiaux et latéraux) et d'autres muscles comme le tenseur du voile du palais et tenseur du tympan (origine de l'expression tendre l'oreille). Le foramen épineux se situe en arrière et latéralement par rapport au foramen oval. Il livre passage à une petite branche du V3 réservée aux méninges et des artères méningées moyennes. L'os temporal contribue à la fosse moyenne, présentant le méat acoustique interne situé au-dessus du foramen déchiré. Il livre passage au nerf VII facial et VIII vestibulo-cochléaire ou acoustique. De part et d'autre du dorsum sellae et situé postéro-latéralement par rapport à la selle turcique se trouve le foramen déchiré. Ce dernier est obturé par une lame cartilagineuse. L'artère carotide interne passe à la face supérieure de ce foramen.

Fosse crânienne postérieure Il s'agit de la plus grande et la plus profonde des 3 fosses crâniennes. Elle est principalement bordée par l'os occipital. Ce dernier possède un plan osseux incliné, le clivus. Postérieurement se trouve la crête occipitale interne qui aboutit à la protubérance occipitale interne. De part et d'autre de la crête occipitale interne se trouvent les fosses cérébelleuses qui contiennent le cervelet. Inférieurement au foramen déchiré se trouve le canal du nerf hypoglosse donnant la motricité de la langue.

\subsection{Face externe de la base du crâne}

\section{Partie antérieure}

Os maxillaire et os palatin On y retrouve le palais dur (palais osseux) formé en avant par les processus palatins des os maxillaires et en arrière par les lames horizontales des os palatins. Sur la ligne médiane de 
l'os maxillaire se trouve la fosse incisive est une dépression située derrière les incisives centrales qui livre passage aux nerfs naso-palatins (branche du nerf V2 maxillaire). Les grands et petits foramina palatins s'ouvrent au niveau de la partie postéro-latérale du palais osseux. Les choanes (ouverture postérieure des fosses nasales) sont séparées par le vomer.

\section{Partie moyenne}

Os sphénoïde L'os sphénoïde est enchâssé entre les os frontal, temporaux et occipital. Il présente un corps et 3 paires de processus : grandes ailes et petites ailes (expansions latérales du corps) ainsi que les processus ptérygoïdes (expansions inférieures de la zone de jonction entre le corps et la grande aile). Chaque processus ptérygoïde se compose d'une lame latérale et une lame médiale, séparées par une fosse ptérygoïdienne. L'os sphénoïde présente les foramina en croissant, mieux visibles sur la face interne du crâne ; néanmoins, le foramen oval (livrant passage au nerf V3 mandibulaire) ainsi que l'épineux (livrant passage à une petite branche du V3 et des vaisseaux méningées) sont bien visibles sur la face inférieure du crâne. Médialement au foramen ovale peut se présenter le foramen de Vésale (foramen Vesalii) aussi livrant passage à la veine émissaire sphénoïdale.

Os temporal L'os temporal est creusé d'une dépression, la fosse mandibulaire (ou cavité glénoïde du temporal) dans laquelle vient se loger le condyle de la mandibule lorsque la bouche est fermée. L'os temporal présente sur sa face inférieure différents foramina : médialement et supérieurement, de chaque côté de la partie basilaire de l'os occipital, se trouve le foramen déchiré ou lacerum, rempli de cartilage sur le vivant. Inféro latéralement au foramen déchiré et supéro médialement au processus stylö̈de se trouve le canal ou foramen carotidien, livrant passage à l'artère carotide interne. Inférieurement au canal carotidien, confinant avec l'os occipital, se trouve le foramen jugulaire, livrant passage à la veine jugulaire interne ainsi que les nerfs crâniens IX glossopharyngien, $\mathrm{X}$ vague, et XI spinal ou accessoire. Le nerf IX glossopharyngien possède cinq fonctions : innervation motrice du muscle stylopharyngien et du constricteur du pharynx supérieur, innervation sensitive la langue postérieurement au V lingual, de la membrane tympanique, le pharynx supérieur et le sinus carotidien, innervation parasympathique de la glande parotide via le ganglion otique. Le nerf $\mathrm{X}$ vague (anciennement nerf pneumogastrique ou entérique) possède principalement une fonction parasympathique destinée au cœur, poumons et tractus digestif ; indirectement et via ses branches laryngées (nerf laryngé supérieur et récurrent) il innerve les muscles du larynx. Le nerf XI spinal ou accessoire est le nerf responsable de l'innervation motrice du muscle sternocléidomastoïdien et du muscle trapèze. Entre le foramen carotidien et le 
foramen jugulaire se situe le foramen livrant passage au nerf tympanique de Jacobson ; il s'agit d'une branche du nerf IX glossopharyngien s'acheminant dans l'os temporal (portion pétreuse) pour former le plexus tympanique, donnant naissance aux nerfs grand et petit pétreux, ainsi que la branche destinée à la cavité tympanique. Le passage osseux (parfois retrouvé comme un simple bord) destiné à ce nerf est parfois dénommé canalicule tympanique de Jacobson. Latéralement au foramen jugulaire, derrière le processus styloïde, se trouve le foramen stylomastoïdien livrant passage au nerf VII facial pour l'innervation motrice des muscles de la face.

\section{Partie postérieure}

Os occipital L'os occipital s'articule en avant avec l'os sphénoïde. Il entoure le foramen magnum par où passent la moelle épinière et ses enveloppes, les artères vertébrales et les artères spinales antérieures et postérieures. La partie basilaire (correspondant au clivus à la face interne de la base du crâne) est le prolongement entre les 2 os temporaux et s'articule avec le sphénoïde en avant. Le clivus peut présenter dans certains cas des variations anatomiques telle que le canalis basilaris medianus (canal traversant le clivus) ou la fossa navicularis, une dépression osseuse se situant sur la partie exocrânienne et pouvant s'abcéder lors d'une infection du pharynx. Ventralement et latéralement par rapport au foramen magnum, on observe deux reliefs osseux importants : les condyles occipitaux par lesquels le crane s'articule avec la 1ère vertèbre cervicale atlas). En avant du condyle occipital et derrière le foramen jugulaire se trouve le canal du nerf hypoglosse, se prolongeant dans la face interne de la base du crâne. Derrière le condyle occipital se trouve le canal condylaire, livrant passage à une veine émissaire.

\section{Dissection cadavérique de la base du crâne}

\section{1 Étapes préalables de dissection cadavérique pour attein- dre la base du crâne}

Lors d'une dissection cadavérique de novo, l'approche de la base du crâne pour la mise en évidence des structures anatomiques y siégeant est secondaire à la dissection antérieure et postérieure des différentes couches cervicales externes à la gouttière trachéo-oesophagienne et la colonne vertébrale cervicale. Le but de ces manoeuvres est in fine de désolidariser la base du crâne du reste du corps pour une étude approfondie.

Dissection antérieure du cou La dissection antérieure du cou se fait par étage. L'incision initiale de la peau est comprise de deux incisions horizontales et d'une médio-sagittale. L'incision horizontale supérieure se fait en 
correspondance de la ligne mandibulaire horizontale, et l'inférieure en regard du bord sternal de la clavicule bilatéralement ; l'incision médio-sagittale sera donc perpendiculaire aux deux horizontales. La profondeur de l'incision doit être minime, puisque le premier étage de dissection est constitué par le muscle platysma (ou peaucier du cou). Le platysma est un muscle aponévrotique tendu irrégulièrement de la mandibule (insertion longitudinale et horizontale supérieurement à la ligne mandibulaire horizontale, inférieurement aux foramens mentonniers) et distalement au niveau du tiers médial de la clavicule, du manubrium sternal, de la première et de la deuxième côte bilatéralement. Le platysma sera incisé inférieurement au niveau des trois insertions distales pour accéder au deuxième étage de la dissection antérieure du cou. Le muscle platysma est innervé par le nerf VII facial via les branches du plexus cervical (branches $\mathrm{C} 1$ et $\mathrm{C} 2$ ). Le deuxième étage de dissection antérieure du cou est constitué du muscle sternocléidomastoïdien bilatéralement et du plexus veineux cervical superficiel. Le sternocléidomastoïdien est tendu du processus mastoïde de l'os temporal et de la ligne nucale supérieure au tiers médial de la clavicule et au manubrium sternal et est innervé par le nerf XI spinal ou accessoire pour une fonction rotatoire interne du cou (le cou est basculé vers l'avant si contraction simultanée des deux muscles). Le réseau veineux superficiel est composé des veines jugulaires antérieures et externes (l'antérieure légèrement parasagittale ainsi que médiale à l'externe). Les deux veines jugulaires de ce plexus forment des anastomoses parfois également avec la veine jugulaire interne plus profondément, mais se jettent le plus souvent dans le confluent jugulo-sous-clavier inférieurement. Le plexus veineux superficiel présente le plus souvent des variantes anatomiques : il peut exister une seule veine jugulaire antérieure, ou bien un décalage latéral des deux paires de veines. Une incision inférieure sera pratiquée en regard du bord claviculaire du muscle ainsi que des veines constituant le deuxième étage de dissection. Après la dissection du deuxième plan, la gaine carotidienne sera visible ; une dissection précoce de cette dernière pourrait entrainer des déchirures, puisque la gaine est dissécable sur toute sa longueur cervicale uniquement après résection des plans musculaires centro-cervicaux. Le troisième étage de dissection est composé des muscles sternohyoïdien et omohyoïdien, du groupe des muscles sous-hyoïdiens ou dépresseurs de l'hyoïde. Le sternohyoïdien est tendu entre l'os hyoïde et le manubrium et est innervé par les branches $\mathrm{C} 1 / \mathrm{C} 2$ du plexus cervical. L'omohyoïdien est tendu entre l'os hyoïde et la scapula et est divisé en deux parties séparées par un tournant tendineux au niveau rétro-claviculaire. La moitié supérieure est innervée par les branches $\mathrm{C} 1 / \mathrm{C} 2$ et la branche inférieure par $\mathrm{C} 2 / \mathrm{C} 3$. Le quatrième étage de dissection est composé des muscles thyro-hyoïdien et sternothyrö̈dien. Le premier est tendu entre le cartilage thyroïde et l'os hyoïde, innervé par C1/C2 et le deuxième entre le manubrium sternal et le cartilage thyroïdien, innervé par C2/C3. Après dissection de cet étage, la gaine carotidienne peut être disséquée. Celle-ci contient antéro-latéralement la veine jugulaire 
interne, postéro-médialement la carotide commune, et postérieurement à ces deux structures le nerf vague. La carotide commune se prolongera au niveau de la base du crâne par la carotide interne pénétrant dans le foramen carotidien, traversant le canal carotidien et gagnant la base interne du crâne par le trou déchiré antérieur. La veine jugulaire interne et le nerf vague gagneront ensemble au niveau de la base du crâne le foramen jugulaire (appelé trou déchiré postérieur au niveau de la face interne de la base du crâne). Une désolidarisation inférieure au niveau du bord supérieur de la clavicule de ces trois structures est utile pour la poursuite de la dissection. La carotide commune ou primitive se divisera au niveau du bord supérieur du cartilage thyroïde en carotide externe ou interne. La carotide interne poursuivra vers l'espace profond de la face rétrostylien avant de se jeter dans le foramen carotidien sur l'os temporal. La carotide externe donnera successivement les artères suivantes : thyroïdienne supérieure, la pharyngienne ascendante, la linguale, la faciale, l'occipitale et l'auriculaire postérieure, avant de se terminer par l'artère maxillaire et temporale superficielle (mnémotechnique : some anatomists like freaking out poor medical students). Au niveau cervical antérieur, le triangle des scalènes sera accessible après la dissection effectuée de l'axe cervical. Le muscle scalène antérieur est tendu entre les processus transverses des vertèbres $\mathrm{C} 3$ à $\mathrm{C} 6$ et le tubercule scalène de la première côte. Il est innervé par les branches $\mathrm{C} 4$ à $\mathrm{C} 7$. Le muscle scalène moyen est tendu entre les processus transverses des côtes C6 à C12 et le tubercule du scalène. Innervation fournie par branches C3 à C7 Le muscle scalène postérieur est tendu entre les processus transverses des côtes C10 à C12 à la surface de la deuxième côte postérieurement à l'insertion du scalène antérieur. Innervation par branches C5 à C7. Le muscle scalène antérieur sert de lit pour la descente du nerf phrénique. Il est postérieur à la veine sous-clavière. Le plexus brachial et l'artère sous-clavières sont situés entre le muscle scalène antérieur et moyen, et sont à désolidariser le plus supérieurement possible pour préserver la dissection et l'étude des parties inférieures du corps (notamment les membres). L'artère sous-clavière donnera naissance aux artères vertébrale, thoracique interne, tronc thyrocervical (se divisant en artère thyroïdienne inférieure, suprascapulaire et cervicale transverse), le tronc costocervical (artère intercostale et artère cervicale profonde), et l'artère scapulaire dorsale. Supérieurement à l'os hyoïde, les muscles du plancher buccal sont observables. Le muscle digastrique est le plus antérieur et composé d'un ventre antérieur, s'insérant dans la fosse digastrique (dépression latérale à l'apophyse génie dans la face interne et inférieure de la ligne mandibulaire horizontale) et d'un ventre postérieur s'insérant sur le processus mastoïde. Les deux ventres du digastriques se rejoignent dans un tendon commun rattaché par une charnière aponévrotique à l'os hyoïde. L'innervation du ventre antérieur est fournie par le nerf V3 mandibulaire et celle du ventre postérieur est fournie par le nerf VII facial. Le muscle stylohyoïdien, tendu depuis le processus stylö̈de de l'os temporal, 
utilise la charnière tendineuse du digastrique pour s'insérer sur l'os hyoïde : le tendon du muscle stylohyoïdien s'insère en fourche sur la charnière. Le muscle stylohyoïdien démarre inférieurement au ventre postérieur du digastrique mais sa pente descendante est bien moins aigue, et les deux muscles forment ainsi une structure en forme de X. Le stylohyoïdien est innervé par le nerf VII facial. Le muscle mylohyoïdien est un lit musculaire pour le reste des muscles du plancher buccal : il est carré et tendu entre la ligne mylohyoïdienne (partie centrale de la ligne mandibulaire horizontale) et l'os hyoïde ; le muscle mylohyoïdien est visible supérieurement au muscle digastrique. L'innervation du muscle mylohyö̈dien est fournie par le nerf V3 mandibulaire. Les muscles géniohyoïdien (tendu entre l'apophyse génie et l'os hyoïde), génioglosse (entre la symphysis menti de l'apophyse génie et la langue), hyoglosse (entre l'os hyoïdien et la langue) et styloglosse (entre le processus styloïde et la langue) sont visibles après désolidarisation de la mandibule, étape fondamentale à la suite de la dissection. Une fois la mandibule retirée via l'articulation temporo-mandibulaire), le larynx peut être réséqué. Celui-ci présente les cartilages épiglotte, thyroïdien, cricoïde et aryténoïdien. Les cordes vocales sont tendues entre le cartilage aryténoïde et thyrö̈de. Les muscles intrinsèques du larynx sont innervés par le nerf laryngé inférieur ou récurrent à l'exception du cricothyroïdien, innervé par la branche externe du nerf laryngé supérieur émanant du nerf $\mathrm{X}$ vague. Les muscles intrinsèques du larynx comprennent également le cricoaryténoïdien postérieur et latéral, l'aryténoïdien transverse et oblique ainsi que le muscle vocalis et thyro-aryténoïdien et sont visibles à la dissection attentive du larynx. Ils prennent leurs insertions selon leurs dénominations respectives. L'aryténoïdien transverse et cricoaryténoïdien latéral causent une adduction des cordes vocales, le muscle cricothyroïdien pousse le cartilage thyroïde vers l'avant et le bas, le cricoaryténoïdien postérieur est abducteur des cordes vocales, le thyro-aryténoïdien et aryténoïdien oblique servent à régler le passage laryngé. Le muscle vocalis règle la tension dans les plis vocaux. Une fois le larynx réséqué antérieurement, la dissection des constricteurs du pharynx (supérieur, moyen, inferieur avec comme origine respectivement les processus ptérygoïdes, le raphé pharyngien et le muscle mylohyoïdien et comme insertion respectivement le fascia pharyngo-basilaire, l'os hyoïde et le cartilage cricoïde, innervés par les nerfs IX glossopharyngien et $\mathrm{X}$ vague), la dissection postérieure du cou peut commencer.

Dissection postérieure du cou La dissection postérieure du cou commence par une résection de la peau selon des axes correspondants à ceux de la dissection antérieure du cou. Une première incision sera effectuée en regard de la ligne nucale supérieure, une incision distale horizontalement reliant les deux acromions bilatéralement. Une troisième incision verticale sera pratiquée médiosagittalement et en bénéficiant comme point de départ 
supérieur la protubérance occipitale externe ou inion. Le premier muscle rencontré après dissection de la graisse sera le trapèze. Le muscle trapèze est tendu entre la ligne nucale supérieure, le ligament nucal et les processus épineux des vertèbres $\mathrm{C} 7$ à $\mathrm{T} 12$ aux acromions scapulaires. Il est innervé par le nerf XI spinal ou accessoire, pour une fonction d'élévation, adduction et dépression de la scapula. Le trapèze peut être désolidarisé au niveau de la ligne nucale supérieure pour préserver une dissection inférieure accessoire au niveau du reste du corps. Après résection du trapèze, le muscle élévateur de la scapula est dissécable. Ce dernier est tendu des processus transverses $\mathrm{C} 1$ à $\mathrm{C} 4$ jusqu'au bord médial de la scapula et bénéficie d'une innervation des branches cervicales $\mathrm{C} 3 / \mathrm{C} 4$ ainsi que du nerf scapulaire dorsal (C4/C5). Le muscle élévateur de la scapula doit être incisé inférieurement pour la future désolidarisation de la tête. Les muscles dentelé postérieur supérieur et rhomboïde mineur ainsi que le reste des muscles postérieurs du cou peuvent être désolidarisés à leurs origines axiales suite à la dissection du muscle élévateur de la scapula. Les muscles seront décrits ici du plus externe au plus interne (en d'autres termes, en ordre de dissection). Le muscle dentelé postérieur supérieur origine des processus épineux $\mathrm{C} 7$ à $\mathrm{T} 4$ et se termine sur l'angle postérieur des cinq premières côtes et bénéficie d'une innervation par les nerfs intercostaux. Le muscle rhomboïde mineur (ou petit rhomboïde) est tendu des processus épineux C6-C7 jusqu'au bord spinal de la scapula. Il est innervé par le nerf scapulaire dorsal (C4/C5). Le muscle splenius capitis débute du ligament nucal et les processus épineux C3 à T3 et se termine sur le processus mastoïde. Il est innervé par les branches C2-C6. Le muscle splenius cervicis débute du ligament nucal et les processus épineux C7-T6 et s'insère sur les processus transverses des vertèbres $\mathrm{C} 1 / \mathrm{C} 3$. Il est innervé par les branches cervicales C2-C6. Le muscle longissimus cervicis débute des processus transverses des six premières vertèbres thoraciques et se termine sur les processus transverses $\mathrm{C} 2$ à $\mathrm{C} 5$. Il est innervé par les branches $\mathrm{C} 1$ à S1. Le muscle iliocostalis débute des angles des côtes 3 à 7 et se termine sur les processus transverses $\mathrm{C} 4$ à $\mathrm{C} 6$. Il est innervé par les nerfs spinaux C5 à C7. Les muscles semispinalis capitis, semispinalis cervicis, et spinalis cervicis s'insèrent des processus transverses de $\mathrm{C} 4$ à $\mathrm{T} 6$ à respectivement la ligne nuchale supérieure, les deux premières vertèbres cervicales et $\mathrm{C} 1$. Une fois ces plans musculaires disséqués, l'axis peut être désolidarisé de C3 pour la dissection profonde de la base du crâne.

Dissection des muscles masticateurs et des espaces profonds de la face La dissection de la face commence par une incision verticale médiosagittale contournant les muscles orbiculaires des yeux et de la bouche. Les muscles nécessaires pour la dissection aisée de la mandibule sont le masséter et le buccinateur. Le masséter peut être disséqué après libération du muscle zygomatique et risorius (la très fréquemment composés de fins faisceaux 
aponévrotiques ; ils seront libérés à leur origine zygomatique puisqu'ils se terminent sur les faisceaux du muscle orbicularis oris) et de la glande parotide située dans la loge parotidienne (faisant partie des espaces profonds de la face), située en pré-auriculaire et contenant les faisceaux du nerf facial. La glande parotide doit être désolidarisée de la face pour la bonne poursuite de la dissection. Le canal de Sténon émanant de la glande parotide sera désolidarisé du muscle masséter et incisé le plus distalement. Le masséter origine de l'arcade zygomatique et se termine sur l'angle de la mandibule ; il est innervé par le nerf V3 mandibulaire. Le muscle buccinateur origine du raphé ptérygomandibulaire et se termine sur les fibres du muscle orbicularis oris. Il est innervé par le nerf buccal émanant du nerf VII facial. Les espaces profonds de la face comprennent l'espace pré-vertébral ou rétro-pharyngé (plan musculaire contenant le muscles longus colli originant de l'atlas et se terminant sur les processus transverses C5 à T3 innervé par $\mathrm{C} 2$ à $\mathrm{C} 6$, et longus capitis originant de la partie basilaire de l'os occipital et se terminant sur les processus transverses C3 à C6 - innervé par $\mathrm{C} 1 / \mathrm{C} 4$ ou $\mathrm{C} 3 / \mathrm{C} 4$ ), la région rétro-stylienne (canal virtuel aérien comprenant le passage des l'artère carotide interne, la veine jugulaire interne et les nerfs IX glossopharyngien, $\mathrm{X}$ vague, XI spinal ou accessoire, et XII hypoglosse sortant par le canal hypoglosse du foramen occipital ; la région rétrostylienne est donc une région de transition), la région parapharyngée (contenant le muscle styloglosse constituant le quart supérieur de la langue, l'artère pharyngienne ascendante branche de la carotide externe et l'artère palatine ascendante, branche de l'artère faciale dérivant de l'artère carotide externe), et la région ptérygo-palatine (permettant la communication entre fosse nasale et fosse intra-temporale et donc l'existence du foramen sphénopalatin livrant le passage à la veine et l'artère sphénopalatine branche distale de l'artère maxillaire). Les muscles ptérygo-palatins latéral et médial débutent respectivement sur les lames ptérygoïdiennes latérale et la fosse ptérygoïdienne ou ptérygo-palatine (entre lame ptérygoïdienne latérale et médiale) et s'insèrent sur l'angle médial de la mandibule. Ils sont innervés par le nerf V3 mandibulaire via le nerf ptérygoïdien latéral et médian. Le muscle tensor veli palatini (tenseur du voile du palais) débute de la lame ptérygoïdienne médiale et s'insère sur l'aponévrose palatine ; il est également innervé par le nerf V3 mandibulaire. Les muscles ptérygoïdiens et tenseur du voile du palais doivent être désolidarisés supérieurement pour poursuivre la dissection de la base du crâne. Une fois toutes ces couches disséquées, la base externe du crâne peut être étudiée. Pour accéder à la face interne de la base du crâne, la calvaria (voute supérieure du crâne, composée des parties supérieures des os frontaux, temporaux et pariétaux par une circonférence passant par la suture lambda) doit être sciée, et les hémisphères cérébraux désolidarisés. 